\title{
Discrete breathers for understanding reconstructive mineral processes at low temperatures
}

\author{
JFR Archilla*, J Cuevas \\ Grupo de Física No Lineal. Universidad de Sevilla. \\ Departamento de Física Aplicada I. ETSI Informática \\ Avda. Reina Mercedes, s/n. 41012-Sevilla, Spain \\ MD Alba, M Naranjo and JM Trillo \\ Departamento de Química Inorgánica. Universidad de Sevilla. \\ Instituto de Ciencia de Materiales de Sevilla. \\ Consejo Superior de Investigaciones Científicas. \\ P.O. Box 874, 41080-Sevilla (Spain)
}

September 20, 2006

\begin{abstract}
Reconstructive transformations in layered silicates need a high temperature in order to be observed. However, very recently, some systems have been found where transformation can be studied at temperatures $600^{\circ} \mathrm{C}$ below the lowest experimental results previously reported, including sol-gel methods. We explore the possible relation with the existence of intrinsic localized modes, known as discrete breathers. We construct a model for nonlinear vibrations within the cation layer, obtain their parameters and calculate them numerically, obtaining their energies. Their statistics shows that although there are far less breathers than phonons, there are much more above the activation energy, being therefore a good candidate to explain the reconstructive transformations at low temperature.
\end{abstract}

Keywords: discrete breathers; reconstructive transformations; intrinsic localized modes

PACS: 63.20.Pw, 63.20.Ry, 63.50.+x, 66.90.+r, 82.20.-w

\footnotetext{
${ }^{*}$ Corresponding author. Email: archilla@us.es
} 


\section{Introduction}

During the last decade, some of the present authors have achieved the synthesis of crystalline high-temperature polymorphs of rare earth (RE) disilicates $\left(\mathrm{RE}_{2} \mathrm{Si}_{2} \mathrm{O}_{7}\right)$ at non-expected temperatures, significantly lower than those previously reported, through a reconstructive process (LTRT) from clay minerals as the silicon source. ${ }^{1-4}$

This finding is of general importance in the development of advanced structural ceramics ${ }^{5}$ or the storage of radioactive wastes ${ }^{3}$ and should allow completion of the available $\mathrm{Si} \mathrm{O}_{2}-\mathrm{RE}_{2} \mathrm{O}_{3}$ phase diagram. ${ }^{6}$ Although no precise explanation has been found up to now, some of the present authors had previously suggested a chimie douce mechanism based on the diffusion of RE ions into the interlayer space of the expandable clay minerals. ${ }^{3,4}$

MacKay and Aubry ${ }^{7}$ have suggested that a possible effect of the existence of localized nonlinear vibrations, named discrete breathers (DBs) could be an apparent violation of Arrhenius' law, i.e., the phenomenon of chemical reactions taking place at much lower temperatures than expected. Although this hypothesis is adventurous, it is worth exploring. Moreover, experimental evidence of DBs has already been found in several systems such as antiferromagnets, ${ }^{8}$ waveguide arrays,${ }^{9}$ molecular crystals ${ }^{10}$ or Josephsonjunctions. ${ }^{11}$ Moving DBs have been proposed as an explanation of dark tracks in muscovite ${ }^{12}$ and there existence in muscovite has just been proven through a sputtering experiment. ${ }^{13}$

With this aim, we have made calculations and shown that the contribution of DBs can provide an interpretation for LTRT in clay minerals. And in order to give experimental support to the hypothesis of DBs, it will be shown that the LTRT phenomenon is not exclusive of expandable clay minerals, as expected by the previously suggested "chimie douce" mechanism, ${ }^{3,4}$ but also extensible to non-expandable layered silicates, such as mica muscovite. The layout of this work consists of: Section 2: Some structural considerations on the reconstructive nature of transformation of layered silicates into disilicates; Section 3: A report on a new experiment on LTRT performed by the authors on mica muscovite; Section 4: Argumentation about the difficulty to explain by the conventional chemical kinetics model the latter experiment; Section 5: Description of an alternative model based on DBs with numerical calculations; Section 6: After a summary of breather statistics theory, the description of our numerical simulations and the consequences on the reaction rate, we conclude with the possibility of explaining, for the first time, the LTRT phenomenon in the synthesis of high-temperature polymorphs of silicates by the contribution of DBs. The article in itself is ended 
with a summary. Two appendices give some detail on phonon and breather statistics respectively.

\section{From layered silicates to disilicate crystal struc- tures}

The synthesis of disilicates from layered silicates, expandable as clay minerals or non-expandable as mica, actually means a reconstructive transformation as shown below.

Layered silicates are made up from two basic building blocks: a sheet of edge-sharing $\left[\mathrm{SiO}_{4}\right]$ units, the tetrahedral sheet, and another one of edgesharing $\left[\mathrm{MO}_{6}\right]$, the octahedral sheet. There are three main groups of layered silicate minerals, according to the combinations of tetrahedral and octahedral sheets: $1: 1,2: 1$ and 2:1:1. In 2:1, one octahedral sheet is sandwiched between the apices of two tetrahedral ones. In these, also called T-O-T silicates, layers are either held together by weak van der Waals forces if they are neutral, or may have cations between them for charge balance if substitutions in either tetrahedral or octahedral sheet result in a net layer charge. The 2:1 layered silicates are classified as trioctahedral or dioctahedral, after the full occupation of the octahedral sheet by $\mathrm{Mg}$ (II) or two thirds by $\mathrm{Al}(\mathrm{III})$. Talc (trioctahedral) and pyrophillite (dioctahedral) are minerals with non-charged layers. In the case of low charge, it results that the clay minerals have the capacity to expand by taking up $\mathrm{H}_{2} \mathrm{O}$ molecules in the interlayer space. For high charge, there is mica: phlogopite (trioctahedral) and muscovite (dioctahedral).

Muscovite is a mica which layer charge comes from the isomorphic substitution of silicon by aluminium in the tetrahedral sheet. ${ }^{14}$ The potassium located in the interlayer space, for charge balance, cannot be hydrated; thus, muscovite does not expand. Its structure is depicted at the left of Fig. 1 At its right, the interlayer space of muscovite is illustrated and it can be observed that both surfaces of the upper and lower tetrahedral sheet are formed by the basal oxygen atoms from the $\left[\mathrm{SiO}_{4}\right]$ tetrahedra, which form a rough hexagonal honeycomb structure. The interlayer balancing sheet is therefore sandwiched in between, potassium occupying the dimples left at the centre of each pair of hexagonal cells. In real crystals, although the onsite potential created by the silicate layer tends to preserve the symmetry, there are always distortions like tetrahedral rotation (the actual situation in muscovite is shown in this figure). Each potassium ion is surrounded by six other potassium ions in the interlayer sheet with a ditrigonal symmetry. 

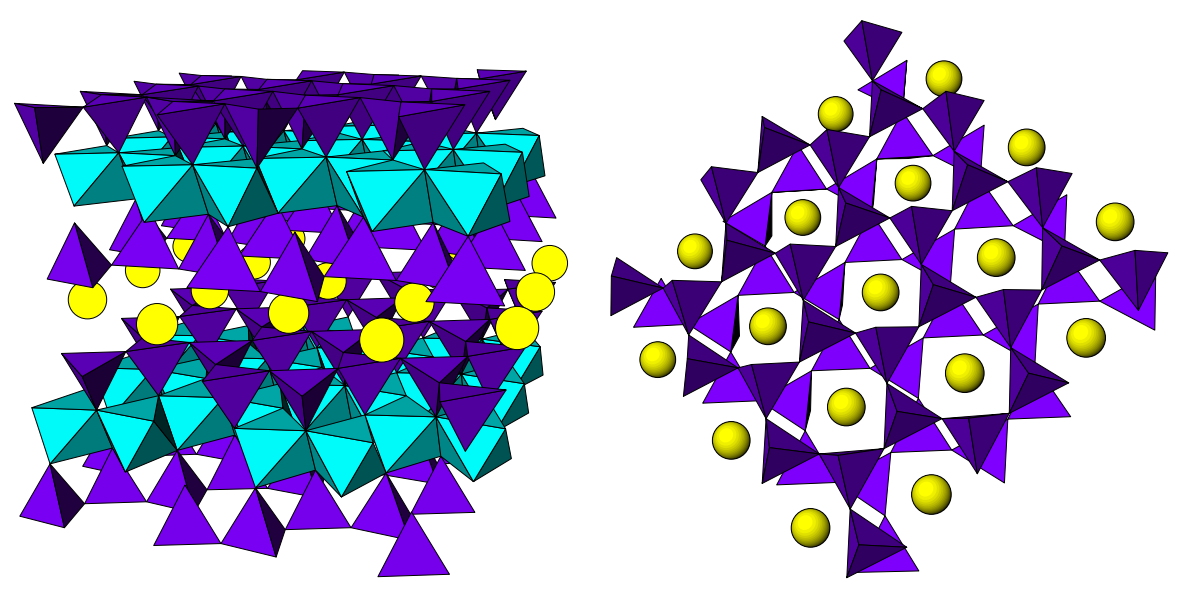

Figure 1: Crystal unit cell of muscovite ICSD 34406. The circles represent the potassium ions forming the interlayer sheet. $(\mathrm{a}=5.19 \AA ; \mathrm{b}=9.02 \AA$; $\mathrm{c}=20.0 \AA ; \beta=95.7^{\circ}$ )

The distance between the potassium and the basal oxygens layers is $1.45 \AA$.

Essentially, in both clay minerals and mica $\left[\mathrm{SiO}_{4}\right]$ tetrahedra are linked into infinite two-dimensional networks by sharing three oxygens. However, disilicates, or pyrosilicates, are the simplest of the condensed forms, where only two tetrahedra share one edge and constitutes the anion $\mathrm{Si}_{2} \mathrm{O}_{7}{ }^{6-}$ (Fig 21). The transformation of any layered-silicate into disilicate involves the rupture of two silicon-oxygen bonds by each $\left[\mathrm{SiO}_{4}\right]$, whatever the reaction mechanism might be, the transformation being reconstructive.

It is well-known that natural pyrosilicates show a wide range of $\mathrm{Si}-\mathrm{O}-\mathrm{Si}$ angle, from $131^{\circ}$ to $180^{\circ},{ }^{15}$ and that the activation energy is reduced if the surface and strain energy terms are diminished by good lattice matching across the interface between the new and parent phase. However, this is not the present case. It rather seems that the disruption of the tetrahedral sheet could be the consequence of localized nonlinear vibration modes as commented in the introduction and explained in the following sections.

\section{Experimental RE-disilicates synthesis}

The method used by us to synthesize RE-disilicates consists of a hydrothermal reconstructive process at low temperatures in an isolated reaction vessel 


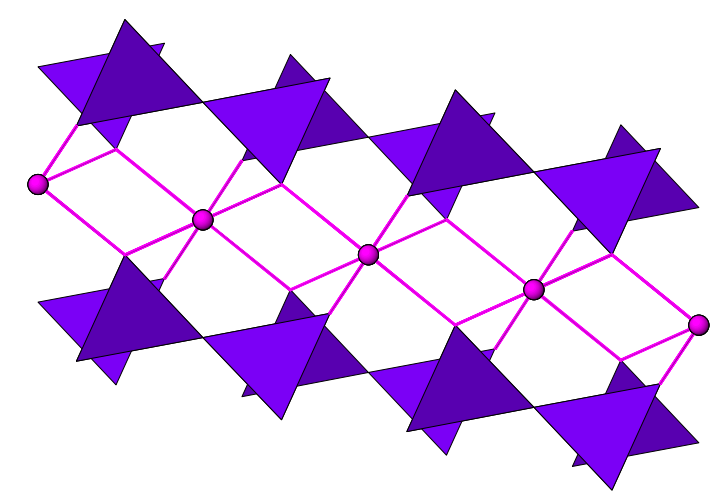

Figure 2: Structure of lutetium disilicate. The circles represent the lutetium ions.

constructed in our laboratory. A layered silicate and an aqueous solution are the silicon and RE(III) sources, respectively. Up to now, a set of expandable clay minerals had been studied, rendering conclusions on the relationship between mineralogical compositions and reactivity. ${ }^{3}$ The reaction temperatures were always below the critical one of water; thus both vapour and liquid phases coexist throughout the whole reaction. Reconstructive structural changes occurring in the layered silicate are always analyzed studying the long-range order by X-ray powder diffraction (XRD), the chemical environment of the main constituent elements of the lattice by magic-angle spinning nuclear magnetic resonance spectroscopy (MAS-NMR) and the microstructural and microchemical composition by electron microscopy (SEM) and by energy-dispersive X-ray (EDX).

As it has been already mentioned, it has been suggested ${ }^{7}$ that DBs might bring about an apparent violation of Arrhenius law, leading to chemical reactions being observable at much lower temperatures than expected. ${ }^{3}$ It has also been suggested that DBs in the interlayer potassium sheet may be responsible for the dark lines observed in crystals of mica muscovite ${ }^{12,16}$ and the existence of moving breathers in mica has been recently proven through a sputtering experiment. ${ }^{13}$ It implies a LTRT process occurring in a nonexpendable layered silicate, which contradicts the mechanism published by some of the present authors to explain the synthesis of RE-disilicates as associated to the capacity for expanding. ${ }^{3}$ In order to confirm experimentally the hypothesis that the LTRT phenomenon is not an exclusive feature of clay minerals, but can also be attributed to non-expandable layered silicates, we 
have performed the hydrothermal synthesis of Lu-disilicate from muscovite, under the same experimental conditions as those used for clay minerals.

When muscovite is hydrothermally treated in stainless steel reactors at $300{ }^{\circ} \mathrm{C}$ for 72 hours with lutetium nitrate $0.05 \mathrm{M}$ solution, it gives rise to $\mathrm{Lu}_{2} \mathrm{Si}_{2} \mathrm{O}_{7}$. Figure 3 shows the XRD diagram of the untreated muscovite. It reveals numerous hkl basal reflections compatible with the $2 \mathrm{M}_{1}$ polytype and a perfect ordering of the layers. After the hydrothermal treatment, the XRD pattern shown in figure $3 \mathrm{~b}$ exhibits a number of specific reflections which are consistent with the development of a new crystalline phase $\mathrm{Lu}_{2} \mathrm{Si}_{2} \mathrm{O}_{7}$ (JCPDS file number 76-1871). The SEM photograph of the mica shows big flakes whereas the sample submitted to hydrothermal treatment, in addition reveals irregular, rough particles, which correspond to $\mathrm{Lu}_{2} \mathrm{Si}_{2} \mathrm{O}_{7}$. The ${ }^{29} \mathrm{Si}$ MAS NMR spectra of both expandable and non-expandable layered silicates show an evolution from $\mathrm{Q}^{3}$ silicon environment to $\mathrm{Q}^{1}$ environment. ${ }^{17}$ This result supports that the LTRT phenomenon is common to expandable and non-expandable layered silicates, both containing an interlayer sheet of cations to balance the layer charge.

Note that although the hydrothermal treatment of layered silicates, muscovite and others, is, effectively, at the origin of the development of a new crystalline phase, as a necessary experimental condition, it is not sufficient. In the case of a silicon source different from a layered silicate, which has been used by the authors for the first time, the process does not occur. An example is illustrated in the Fig. 4 This figure shows the ${ }^{29} \mathrm{Si}$ MAS NMR of $\mathrm{SiO}_{2}$ submitted to a hydrothermal treatment at $300{ }^{\circ} \mathrm{C}(\mathrm{b})$ and the typical spectrum of the lutetium disilicate (a). The signal of the $\mathrm{SiO}_{2}$ after hydrothermal treatment remains in the chemical shift range typical of $\mathrm{Q}^{4}$ environment (typical of $\mathrm{SiO}_{2}$ ), which is clearly different for $\mathrm{Q}^{1}$ environment (typical for the new phase $\mathrm{Lu}_{2} \mathrm{Si}_{2} \mathrm{O}_{7}$ ). Thus, it demonstrates that under hydrothermal condition, $\mathrm{SiO}_{2}$ does not transform in a new phase.

\section{The conventional chemical kinetics approach}

Transformation processes of minerals in which there is a major reorganization with bonds and, even, change in the chemical composition are classified as reconstructive. These obey mechanisms which involve very high activation energies when the rupture of strong bonds are involved. Synthesis of RE-disilicates from layered silicates requires the rupture of silicon-oxygen bonds, which are considerably stronger than the bonds between any other element and oxygen. Silicate minerals make up the vast majority of rocks 


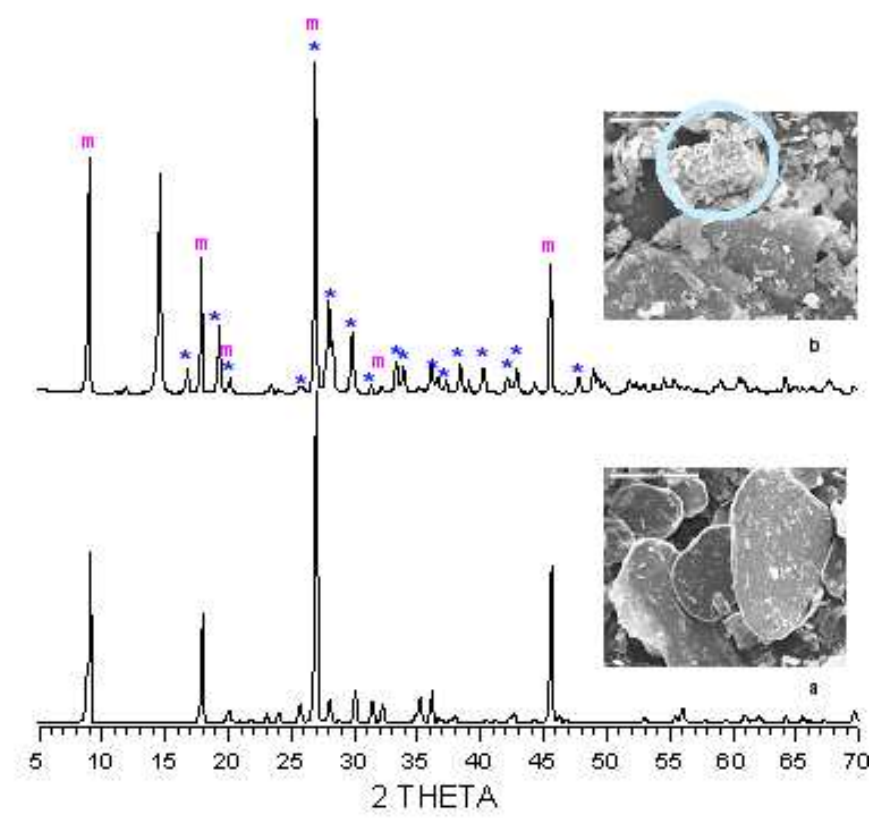

Figure 3: XRD pattern and SEM micrography of untreated (a) and hydrothermally treated (b) muscovite. $\mathrm{m}=$ muscovite, ${ }^{*}=\mathrm{Lu}_{2} \mathrm{Si}_{2} \mathrm{O}_{7}$. The composition of the rough particle inside the circle is compatible with $\mathrm{Lu}_{2} \mathrm{Si}_{2} \mathrm{O}_{7}$

and their reconstructive transformation processes show activation energies as high as $200 \mathrm{~kJ} / \mathrm{mol}$ or even higher. ${ }^{18}$ Therefore, these transformations can be observed in silicate-based minerals at temperatures higher than approximately $1000{ }^{\circ} \mathrm{C}$ and are apparently impossible at lower ones.

It is well known that the over-all effect on temperature on the reaction rate constant $k$ is expressed by Arrhenius law:

$$
k=A \exp \left(-E_{\mathrm{a}} / R T\right)
$$

where $A$ and $E_{\mathrm{a}}$ are the frequency factor and the activation energy, respectively. According to it, and without any further insight into the mechanism, the rate of a reaction is about $10^{9}$ times faster at $1000{ }^{\circ} \mathrm{C}$ than it is at $300{ }^{\circ} \mathrm{C}$ for an activation energy of $200 \mathrm{~kJ} / \mathrm{mol}$.

It can be shown that the second parameter in the Arrhenius law, namely, the frequency factor $A$, does not support LTRT synthesis of RE-disilicates as well. Usually, reaction rates are described within the frame of the quasiequilibrium activated state model. ${ }^{19}$ According to the latter, the necessary 


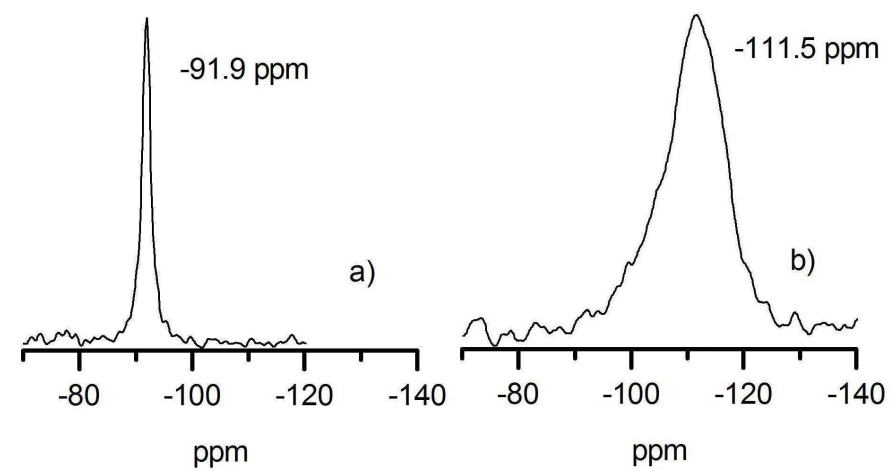

Figure 4: ${ }^{29} \mathrm{Si}$ MAS NMR spectra: a) $\mathrm{Lu}_{2} \mathrm{Si}_{2} \mathrm{O}_{7}$ b) $\mathrm{SiO}_{2}$ treated with $50 \mathrm{ml}$ of $\mathrm{Lu}\left(\mathrm{NO}_{3}\right)_{3}$ at $300{ }^{\circ} \mathrm{C}$

condition for a transformation to take place at a measurable rate is that a sufficient number of atoms have enough energy to achieve the transition state. This energy is supplied by thermal fluctuations. The rate of reaction is then simply the number of activated complexes passing per second over the potential barrier.

Applying the conventional transition state (activated complex) theory ${ }^{20-25}$ to our case, the simplest formulation of the mechanism can be cast in the following form:

$$
\begin{aligned}
\mathrm{S} \text { (layered silicate) }+\mathrm{RE}(\mathrm{III})(\mathrm{aq}) & \leftrightarrow[\mathrm{S}-\mathrm{RE}(\mathrm{III})]^{*} \\
{[\mathrm{~S}-\mathrm{RE}(\mathrm{III})]^{*} } & \rightarrow \mathrm{RE}_{2} \mathrm{Si}_{2} \mathrm{O}_{7}
\end{aligned}
$$

The rate constant $k$ for the reaction can be derived by assuming that the transition state (or activated complex) is in equilibrium with the reactants. If $\mathrm{C}^{*}$ represents the concentration of the transition state then the equilibrium constant is:

$$
K^{*}=\frac{\mathrm{C}^{*}}{[\mathrm{RE}(\mathrm{III})]}
$$

The rate constant $k$ and the equilibrium constant $K^{*}$ are related by the Eyring equation:

$$
k=\nu K^{*}
$$

with $\nu=\mathrm{k}_{\mathrm{B}} T / \mathrm{h}$, h being the Planck constant.

To be precise, the rhs of the above expression should be multiplied by a factor $\eta$, the transmission coefficient, which is the probability that the complex will dissociate into products instead of back into reactants. For most 
reactions $\eta$ is between 0.5 and $1.0{ }^{19}$ Through a thermodynamic formulation of $K^{*}$, it results:

$$
\begin{aligned}
k= & \nu \eta \exp \left(-\Delta G^{0 *} / \mathrm{R} T\right)= \\
& \nu \eta \exp \left(\Delta S^{0 *} / \mathrm{R}\right) \exp \left(-\Delta H^{0 *} / \mathrm{R} T\right)=A \exp \left(-E_{\mathrm{a}} / R T\right),
\end{aligned}
$$

where the superindex ${ }^{0}$ stands for normal conditions. In liquid and solid systems, the $p \Delta V^{0 *}$ term is negligible and $\Delta H^{0}=\Delta E^{0 *}=E_{\mathrm{a}}$.

At $300^{\circ} \mathrm{C}$ we have calculated the factor $A$ by substitution of all the parameters in the above expression and it takes the usual value $10^{13}-10^{14}$ $\mathrm{s}^{-1}$ for a first order reaction. It does not explain the observation of LTRT synthesis of RE-disilicates as previously concluded from $E_{\mathrm{a}}$.

It is well known that, in parallel to the reorganization of the clay, there must be nucleation of $\mathrm{RE}_{2} \mathrm{Si}_{2} \mathrm{O}_{7}$ crystals and that the activation energy is reduced if the surface and strain energy terms are diminished by good lattice matching across the interface between the new and parent phase. ${ }^{4}$ However, this is not the present case. It rather seems that the disruption of the tetrahedral sheet is the consequence of localized nonlinear vibration modes, as suggested in Ref. 7. If the vibration modes were delocalized, the relationship shown in Ref. 18 between bond angle, Si-O distance and free energy would be incompatible with an appreciable parent structure-directing character.

Ytrium disilicate $\left(\mathrm{Y}_{2} \mathrm{Si}_{2} \mathrm{O}_{7}\right)$ has four polymorphs, namely $y, \beta, \gamma$ and $\delta$. Our LTRT synthesis from the two layered silicates saponite and laponite have shown a structure-directing character of the parent clay by only giving $y-\mathrm{Y}_{2} \mathrm{Si}_{2} \mathrm{O}_{7}$ and $\delta-\mathrm{Y}_{2} \mathrm{Si}_{2} \mathrm{O}_{7}$, the lower and higher temperature polymorphs. ${ }^{4}$ The relative position of the two tetrahedra in the disilicate unit of the $y$ and $\gamma$ - polymorphs are similar to their position in the tetrahedral sheet of saponite and laponite, which is $\sim 141^{\circ}$. The Si-O-Si bond angles in $\mathrm{Y}_{2} \mathrm{Si}_{2} \mathrm{O}_{7}$ polymorphs are $134^{\circ}(y), 180^{\circ}(\beta), 170^{\circ}(\gamma)$ and $158^{\circ}(\delta)$. The percentages of $y$ - and $\delta$-phases in the cases of saponite and laponite are explained in Ref. 4 as related with the presence of $\mathrm{Al}(\mathrm{III})$ in the precursor framework. Moreover, the importance of maintaining the local Si-O-Si bond angle of precursor structure is shown by the fact that the $\delta$-polymorph has been synthesized at more than $365^{\circ} \mathrm{C}$ below the stability range shown in the phase diagram:

$$
\begin{array}{ll}
\mathrm{y}-\mathrm{Y}_{2} \mathrm{Si}_{2} \mathrm{O}_{7} \rightarrow \beta-\mathrm{Y}_{2} \mathrm{Si}_{2} \mathrm{O}_{7} & 1050(50){ }^{\circ} \mathrm{C} \\
\beta-\mathrm{Y}_{2} \mathrm{Si}_{2} \mathrm{O}_{7} \rightarrow \gamma-\mathrm{Y}_{2} \mathrm{Si}_{2} \mathrm{O}_{7} & 1350(50){ }^{\circ} \mathrm{C} \\
\beta-\mathrm{Y}_{2} \mathrm{Si}_{2} \mathrm{O}_{7} \rightarrow \delta-\mathrm{Y}_{2} \mathrm{Si}_{2} \mathrm{O}_{7} & 1500(50){ }^{\circ} \mathrm{C}
\end{array}
$$


At the present stage of DBs application to understanding the LTRT phenomenon, other experimental results already published by our group, such as the influence of isomorphic substitution in clay minerals on the reactivity of the latter, are still awaiting their explanation. We hope that the further work on DB in solid state physics will permit to interpret all those experimental results in the near future.

\section{Discrete breathers model for potassium vibra- tions}

As commented above, it has been predicted that discrete breathers could bring about an increase of the reaction rate. ${ }^{7}$ They are localized vibrational modes in networks of nonlinear oscillators, which have been thoroughly studied in the last years. ${ }^{26-28}$ They have been observed in experiments ${ }^{8-11}$ and are thought to play an important role in DNA denaturation. ${ }^{29}$ They were also suggested to be responsible for dark lines in crystals of muscovite ${ }^{12,16}$ and their existence demonstrated in muscovite through a sputtering experiment. ${ }^{13}$

In this paper we have considered breathers for the out-of-plane movements of the potassium cations, i.e. the movements transversal to the cation layer. This layer is considered to be a 2D triangular lattice, as Fig. 5 shows. The Hamiltonian is given by:

$$
H=\sum_{\vec{n}}\left(\frac{1}{2} m \dot{u}_{\vec{n}}^{2}+V\left(u_{\vec{n}}\right)+\frac{1}{2} \kappa \sum_{\vec{n}^{\prime} \in N N}\left(u_{\vec{n}}-u_{\vec{n}^{\prime}}\right)^{2}\right),
$$

where $m=39.1 \mathrm{amu}$ is the mass of a potassium cation, $\kappa$ is the elastic constant of the cation-cation bond, $V\left(u_{\vec{n}}\right)$ is an on-site potential, and the second sum is extended to the nearest-neighbours, as indicated in Fig. 5 . The value of $\kappa$, i.e. the elastic constant for potassium-potassium bond stretching is $10 \pm 1 \mathrm{~N} / \mathrm{m}$, after Ref. 30 .

A band at $143 \mathrm{~cm}^{-1}$ is identified in Ref. 31 with the $\mathrm{K}^{+}$vibration perpendicular to the $\mathrm{K}^{+}$-plane in spectra from 30 to $230 \mathrm{~cm}^{-1}$. We have performed infrared spectra in CNRS-LADIR ${ }^{1}$ above $200 \mathrm{~cm}^{-1}$. There appear bands at 260,350 and $420 \mathrm{~cm}^{-1}$ which we assign tentatively to higher order transitions of the same vibration. Using standard numerical methods to solve the Schrödinger equation for the $\mathrm{K}^{+}$vibrations with a potential composed of the linear combination with three gaussians and a polynomial of degree six,

\footnotetext{
${ }^{1}$ Laboratoire de Dynamique, Interactions et Réactivité at CNRS-Thiais, Paris
} 


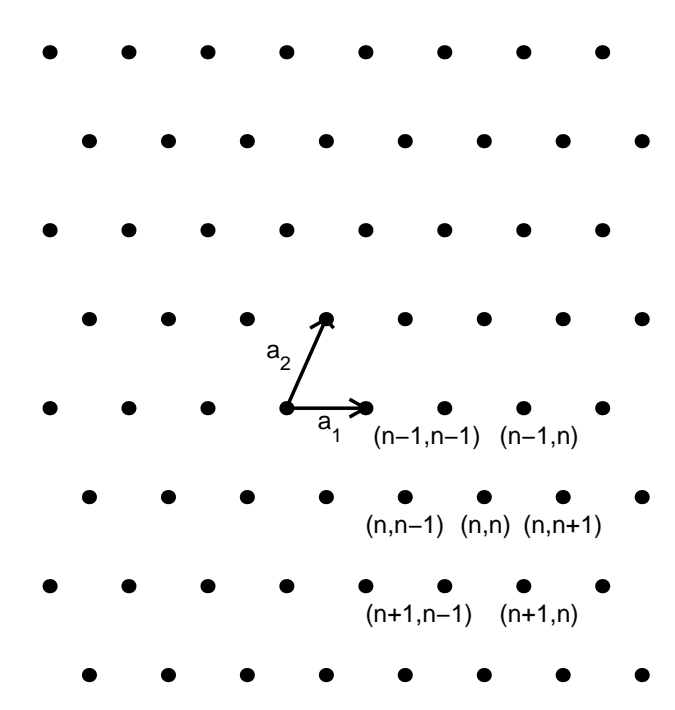

Figure 5: Scheme of the 2D lattice used for discrete breather calculation. $\mathrm{a}_{1}$ and $\mathrm{a}_{2}$ are the base vectors.

with adjustable parameters, we have been able to find a suitable potential that fits these bands and their intensities. It is given by:

$$
V(u)=D\left(1-\exp \left(-b^{2} u^{2}\right)\right)+\gamma u^{6},
$$

with $D=453.11 \mathrm{~cm}^{-1}, b^{2}=36.0023 \AA^{-2}$ and $\gamma=49884 \mathrm{~cm}^{-1} \AA^{-6}$. To completely define the potential, the limitation to the $\mathrm{K}^{+}$displacement due to the muscovite structure has also been taken into account. Fig. [6 shows the adjusted potential together with the observed bands.

The nonlinearity of the on-site potential allows the existence of intrinsic localized modes or discrete breathers apart from low-amplitude linear modes. ${ }^{7}$ These localized solutions exist as long as no integer multiple of their frequency resonates with the frequency of a linear mode and can be obtained numerically with machine precision. They are calculated using procedures based on the anti-continuous limit $(\kappa=0),{ }^{32}$ which consists of calculating an orbit of an isolated oscillator submitted to the potential $V(u)$ with a fixed frequency $\nu_{\mathrm{b}}$, and using this solution as a seed for calculating the solution of the full dynamical equations $(\kappa \neq 0)$ through a continuation method.

In principle, we have considered excitations in the soft part of the on-site potential (the energy of an isolated oscillator decreases with the frequency) 


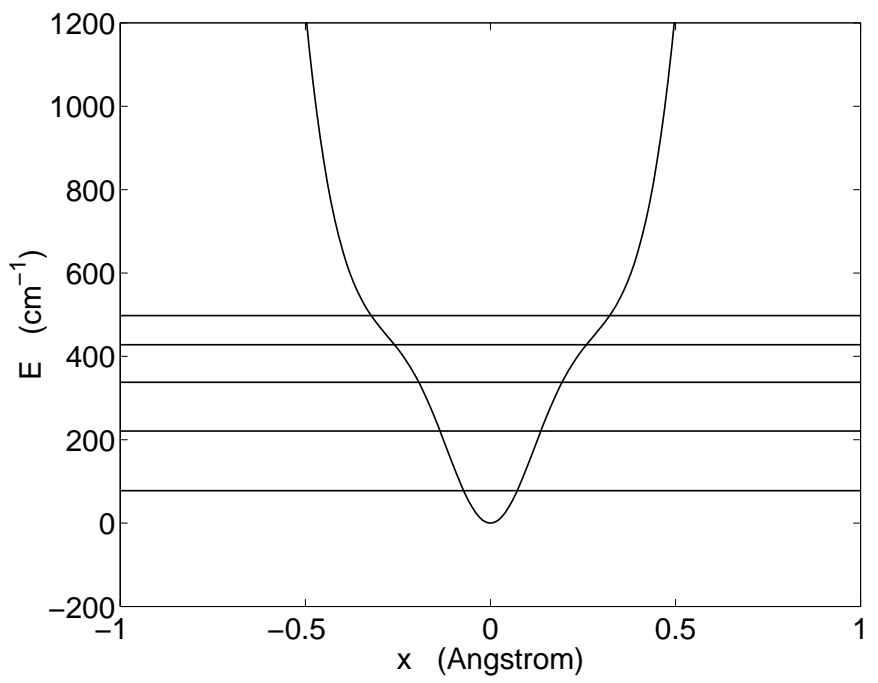

Figure 6: Numerically calculated on-site potential for the potassium vibration based in the observed bands (horizontal lines)

because this is the region that fits the experimental data. In this case, the frequency of a breather $\nu_{\mathrm{b}}$ is below the linear modes band and none of its harmonics coincide with the frequency of a linear mode for a given value of $\kappa$.

The linear modes are solutions of the equations:

$$
\begin{array}{r}
m \ddot{u}_{n, n^{\prime}}+m \omega_{\mathrm{o}}^{2} u_{n, n^{\prime}}-\kappa\left(u_{n, n^{\prime}-1}+u_{n, n^{\prime}+1}+u_{n-1, n^{\prime}-1}+u_{n-1, n^{\prime}}+\right. \\
\left.u_{n+1, n^{\prime}-1}+u_{n+1, n^{\prime}}-6 u_{n, n^{\prime}}\right)=0,
\end{array}
$$

with $\omega_{\mathrm{o}}^{2}=V^{\prime \prime}(0) / m$. Then, the linear modes spectrum is the following:

$$
\omega^{2}=\omega_{\mathrm{o}}^{2}\left[1+4 C\left(\sin ^{2}\left(\theta_{1} / 2\right)+\sin ^{2}\left(\theta_{2} / 2\right)+\sin ^{2}\left(\theta_{2} / 2-\theta_{1} / 2\right)\right)\right],
$$

with $C=\kappa /\left(m \omega_{\mathrm{o}}^{2}\right) \approx 0.15$ and $\theta_{i} \in[-\pi, \pi]$. Thus, the frequency of the linear modes lies in the interval $\nu \in \nu_{0}(1, \sqrt{1+9 C})$, with $\nu_{0}=\omega_{\mathrm{o}} /(2 \pi)=$ $167.50 \mathrm{~cm}^{-1}$. In consequence, no multiple of $\nu_{\mathrm{b}}$ must lie in this band.

Fig. 17 shows the profiles of two breathers with different frequencies and Fig. 8 shows the dependence of the breather energy with respect to the frequency. We can observe that there is a minimum energy $\Delta \sim 23 \mathrm{~kJ} / \mathrm{mol}$ for breather creation as it is known to happen for $2-\mathrm{D}$ and $3-\mathrm{D}$ breathers. ${ }^{33}$ 
These breathers are single symmetric breathers, but there are many other different types, as breathers with different symmetries, multibreathers, breathers above the phonon band, which will have different energies, range of existence and probability of excitation. For example, symmetric single breathers with frequencies above the phonon band have energies between 240 and $500 \mathrm{~kJ} / \mathrm{mol}$. The obtention of a complete description for all the different breather types and their ranges of existence and stability is a long task that we do not pursue here.
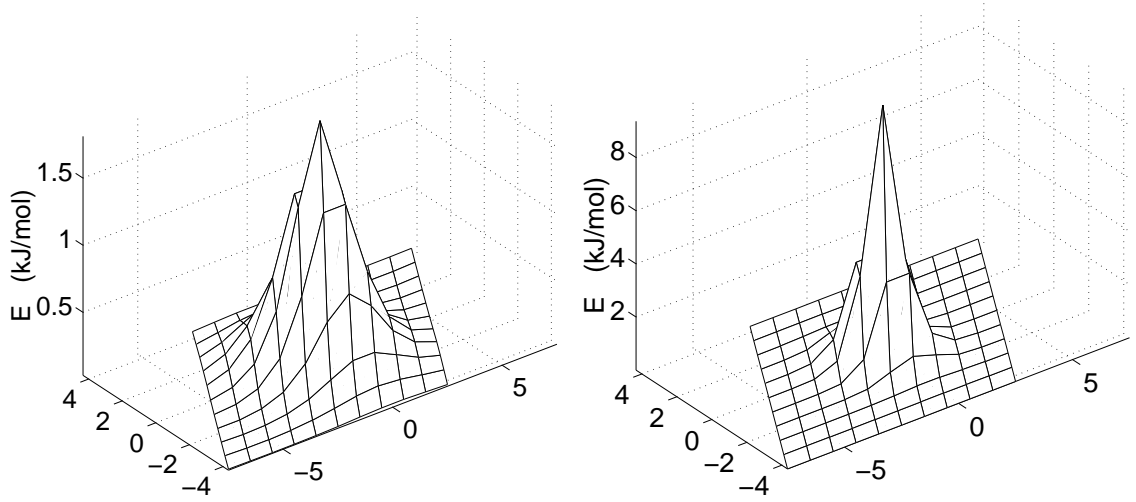

Figure 7: (Left) Energy density profile for a breather with frequency $\nu_{\mathrm{b}}=$ $0.97 \nu_{0}$ and energy $25.6 \mathrm{~kJ} / \mathrm{mol}$. (Right) The same but for a breather with frequency $\nu_{\mathrm{b}}=0.85 \nu_{0}$ and energy $36.3 \mathrm{~kJ} / \mathrm{mol}$. Notice that the localization is higher for the lower frequency. The $x$ and $y$ coordinates are in lattice units of $5.2 \AA, \nu_{0}=167.5 \mathrm{~cm}^{-1}$.

\section{Breather statistics and its effect on the reaction rate}

\subsection{Breather statistics theory}

The effect of the temperature on the reaction rate constant $k$ is given by the Arrhenius law $k=A \exp \left(-\beta E_{\mathrm{a}}\right)$, with $\beta=1 / \mathrm{R} T$. The Boltzmann factor $\exp \left(-\beta E_{\mathrm{a}}\right)$ represents the fraction of vibrational modes that are able to deliver the activation energy $E_{\mathrm{a}}$ for the reaction to proceed. The frequency factor $A$ depends on the reaction kinetics and although it is difficult to 


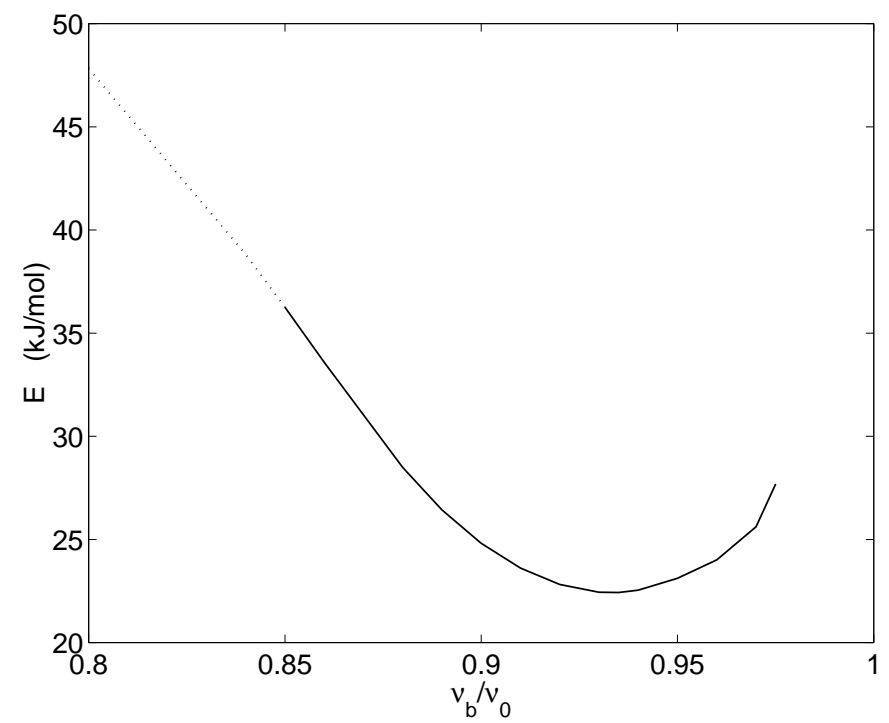

Figure 8: Dependence of the breather energies with the relative frequency for symmetric single breathers with frequencies below the phonon band. The dashed line indicates that breathers are unstable. $\nu_{0}=167.5 \mathrm{~cm}^{-1}$.

calculate the estimation given above is $10^{13}-10^{14} \mathrm{~s}^{-1}$, or the order of magnitude of most chemical reactions.

The statistics of breathers is not at all completed and a full statistical analysis of muscovite and the reaction kinetics is a formidable task. Here we use the theory developed for 2-D breathers by Piazza et $a l^{34}$ which is described in the first subsection of Appendix $\mathbb{B}$, with some modifications in subsection B.2. Note that this theory is not deduced from first principles but it is based in heuristic reasoning. It has to be considered a reasonable approximation justified by its good accuracy with numerical simulations. Here we summarize the main results that are useful to us:

a) The probability density $P_{\mathrm{b}}(E)$, defined so as $P_{\mathrm{b}}(E) \mathrm{d} E$ is the probability of existence or the mean fraction of breathers with energies between $E$ and $E+\mathrm{d} E$, is given by

$$
P_{\mathrm{b}}(E) \mathrm{d} E=\frac{\beta^{z+1}}{\Gamma(z+1)}(E-\Delta)^{z} \exp [-\beta(E-\Delta)],
$$

with $\Gamma(z+1)$, the gamma function, and $z$, a parameter which does not depend on the energy. The cumulative probability, $C_{\mathrm{b}}(E)$ or mean fraction 
of breathers with energy above or equal to $E$ is given by:

$$
C_{\mathrm{b}}(E)=\Gamma(z+1)^{-1} \gamma(z+1, \beta(E-\Delta)),
$$

where $\gamma(z, \epsilon)$ is the incomplete gamma function, defined as $\int_{\epsilon}^{\infty} y^{z-1} \exp (-y) \mathrm{d} y .{ }^{35}$

b) A population of large breathers tends to develop with mean energy $\langle E\rangle=\Delta+(z+1) \mathrm{k}_{\mathrm{B}} T$ and maximum probability for $E\left(P_{\max }\right)=\Delta+z \mathrm{k}_{\mathrm{B}} T$.

Fig. 11] shows the probabilities and cumulative probabilities of phonons and breathers, the latter for $\Delta=20 \mathrm{~kJ} / \mathrm{mol}$ and $z=2$. Note that for large energies of the order of the activation energies, $C_{\mathrm{b}}(E)$, although small, is several orders of magnitude larger than $C_{\mathrm{ph}}(E)$. The drawback is that the number of breathers per site is much smaller than the number of phonons, with typical values of $10^{-3}$ obtained in numerical simulations.

This theory can be modified to take into account that some breather types have an upper limit for their energies, due to bifurcations or instabilities as explained in B.1. The modified probability densities and cumulative probabilities are given in Eqs. (B-6 B-7).

\subsection{Numerical simulations}

We have performed a number of numerical simulations with the following procedure: a) A network of $50 \times 50$ oscillators is given a number of times (about 500) the same energy with different random distributions of velocities; b) They are left evolve a sufficiently long time so as they attain states of thermal equilibrium, for which the mean temperature for all the simulations is calculated; c) The networks are cooled by adding a dissipative term to the dynamical equations for the oscillators at its borders until the energy stops diminishing; d) The number of breathers is counted and their energies are calculated. The number of breathers divided by the number of sites and the number of simulations gives the mean number of breathers $\left\langle n_{\mathrm{b}}\right\rangle$.

Note that the temperature can not be fixed from the outcome. For some energy we obtain a temperature $T_{C}=280.25 \mathrm{C}$, approximately the experimental one, and the data given here correspond to that case. We obtain a mean number of breathers per site $\left\langle n_{\mathrm{b}}\right\rangle=0.92 \times 10^{-3}$, with a mean energy $\langle E\rangle \sim 70 \mathrm{~kJ} / \mathrm{mol}$ which would correspond to $z=9.19$ at the experimental temperature. However, the probability density $P_{\mathrm{b}}(E)$ does not correspond to a curve as the one given by Eq. (10) and plotted in Figure 111 because it is a curve with several maxima and minima (see Fig. 9). The reason is that with the numerical simulations, we obtain all types of multibreathers and single breathers with different symmetries and not only the symmetric, 
single, exact breathers obtained numerically in the previous section, which are continuation from a single excited oscillator at the anticontinuous limit.

Therefore, the numerical curve $P_{\mathrm{b}}(E)$ can be seen as a numerical spectrum for the different breather energies and forms of vibration. The ideal objective, as with other types of spectra, would be to know each type of breather, with its dispersion curve $E\left(\nu_{\mathrm{b}}\right)$, and the relative probability of its appearance and to be able to reproduce exactly the numerical spectrum.

In principle, all the breather types could be obtained exactly with different conditions at the anticontinuous limit with different frequencies and by path continuation by changing the frequency and studying the different branches at the possible bifurcations. This is a long and difficult task that we do not pursue here. Instead, we try to fit approximately the numerical $P_{\mathrm{b}}(E)$, with a small number of breather types, each one characterized by its minimum energy $\Delta$, maximum energy $E_{\mathrm{M}}$, which can be $\infty$, and parameter $z$ (see B.1 and B.2), each breather type with a different probability to occur. In this way, we know that we cannot fit exactly the numerical spectrum because we are most probably substituting a number of breather types with an average one. In any case, our numerical $P_{\mathrm{b}}(E)$ is also an approximation, as we would need a extremely large number of simulations to obtain the actual curve.

Note that the breather spectrum will not appear in an experimental one for three reasons: a) The number of breathers is about $10^{-3}$ the number of phonons, and the spectrum is basically dominated by the one-phonon transitions; b) Breathers are localized and, therefore, they cannot be excited by infrared, raman of neutron spectra. ${ }^{36}$

Figures 9 and 10 show the numerical and theoretical, probability densities and cumulative probabilities, respectively. The parameters of the breathers are:

$\begin{array}{ccccccc}\Delta(\mathrm{kJ} / \mathrm{mol}) & 23.9 & 36.6 & 41.4 & 62.2 & 67.3 & 82.9 \\ z & 1.50 & 1.17 & 3.00 & 0.52 & 2.07 & 1.80 \\ \left.E_{\mathrm{M}}(\mathrm{kJ} / \mathrm{mol})\right) & - & 46.9 & - & - & - & 94.4 \\ \text { Probability } & 0.103 & 0.026 & 0.281 & 0.097 & 0.202 & 0.290\end{array}$

\subsection{Effect on the reaction rate}

According to Ref. 18 the lowest estimates of the activation energy for a reconstructive transformation as the one described above are $E_{\mathrm{a}}=100-$ $200 \mathrm{~kJ} / \mathrm{mol}$. Let $n_{\mathrm{ph}}\left(E_{\mathrm{a}}\right) \simeq \exp \left(-\beta E_{\mathrm{a}}\right)$ and $n_{\mathrm{b}}\left(E_{\mathrm{a}}\right)$ be the mean number of phonons and breathers, respectively, per site, with energies $E \geq E_{\mathrm{a}}$. Then, $n_{\mathrm{b}}\left(E_{\mathrm{a}}\right)=\left\langle n_{\mathrm{b}}\right\rangle C_{\mathrm{b}}\left(E_{a}\right)$, with $\left\langle n_{\mathrm{b}}\right\rangle \sim 0.92 \cdot 10^{-3}$, the mean number of 


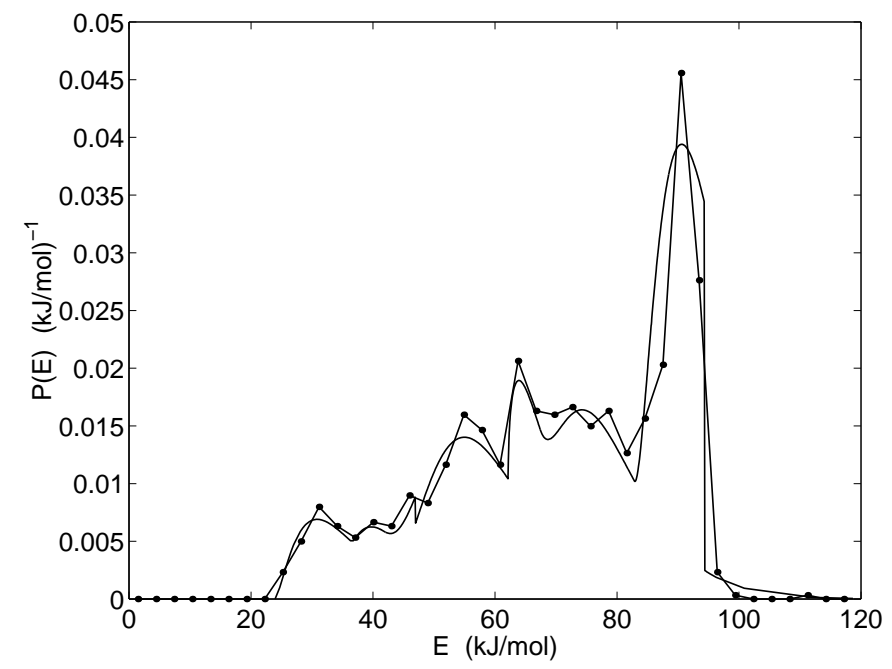

Figure 9: Breathers spectra, i.e., breather probability densities $P_{\mathrm{b}}(E)$ obtained numerically (line with dots) and theoretically (continuous line). The latter is obtained by considering six different types of breathers. See text.

breathers per site obtained numerically, and $C_{\mathrm{b}}\left(E_{a}\right)$, the cumulative probability described in the previous subsection, obtained with six different types of breathers in order to fit the numerical probability density. The ratio of the number of breathers to phonons becomes $n_{\mathrm{b}}\left(E_{\mathrm{a}}\right) / n_{\mathrm{ph}}\left(E_{\mathrm{a}}\right) \sim 10^{4}-10^{5}$.

The reaction rate constant with breathers would be $k_{\mathrm{b}}=A_{\mathrm{b}} n_{\mathrm{b}}\left(E_{\mathrm{a}}\right)$, with $A_{\mathrm{b}}$, the frequency factor for breathers $A_{\mathrm{b}}$, which should be different from $A$, the frequency factor for phonons. We will assume that $A_{\mathrm{b}}=A$ for the purpose of comparison. The ratio of reaction rates for breathers and phonons would be $k_{\mathrm{b}} / k=n_{\mathrm{b}}\left(E_{\mathrm{a}}\right) / n_{\mathrm{ph}}\left(E_{\mathrm{a}}\right) \sim 10^{4}-10^{5}$ for $E_{\mathrm{a}}=100-200$ $\mathrm{kJ} / \mathrm{mol}$. In other words, as the three days experimental time leads to about $30 \%$ of the transformation performed, the time without breathers to obtain the same result, would be $10^{4}-10^{5}$ times larger and, thus, completely unobservable.

Two factors are likely to increase further the reaction rate with breathers. First, since a discrete breather is strongly localized, it seems much more capable of delivering the energy for breaking a Si-O bond, which implies that $A_{\mathrm{b}}$ should in effect be much larger than $A$. Second, for larger systems than the one used in our simulations, the fluctuations may have larger energies and, therefore, excite other types of breathers with higher energies, as, for 


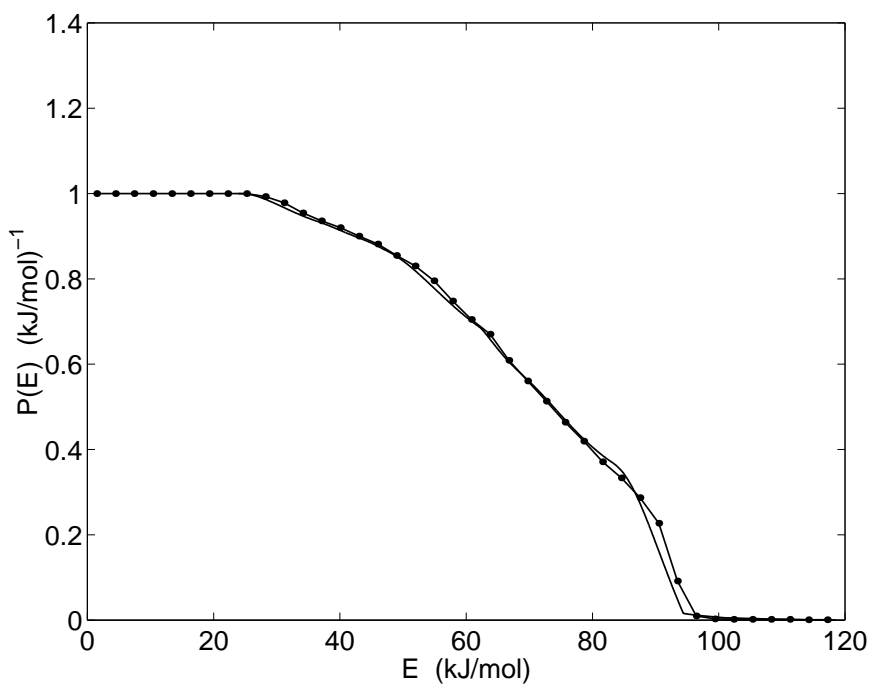

Figure 10: Breather cumulative probabilities $C_{\mathrm{b}}(E)$ obtained numerically (line with dots) and theoretically (continuous line). The latter considering six different types of breathers. See text.

example, breathers with frequencies above the phonon band, which have energies between 240 and $500 \mathrm{~kJ} / \mathrm{mol}$. These breathers would increase the fraction of breathers above the activation energy and therefore the reaction rate.

\section{Summary and conclusions}

Low temperature reconstructive transformations (LTRT) have been achieved in layered silicated by some of the authors at temperatures about $600^{\circ} \mathrm{C}$ lower than previously reported. This is a phenomenon for which there is presently no plausible explanation since the bonds involved are the same as in other transformations. New experiments performed by some of the authors on mica muscovite, a non-expandable silicate have discarded their previous hypothesis of LTRT been caused by the expansion of the intersheet layer.

We have constructed a model for breathers in the cation later, for which we have obtained reasonable parameters, and with a mixture of numerics and theory we have estimated their effect in the reaction rate. The results are 
that they would increase enormously the reaction rate and, thus, explain the observed LTRT. This can be easily explained by the fact that, although there are much less breathers than phonons, there are many more with energies above the expected activation energy. Certainly, the statistical theory of breathers is only an approximation, and the numerics cannot be precise at larger energies for which there are so few breathers, except if an enormous number of simulations could be performed. However, an established fact in breather theory is that large breathers have longer life time than small ones and thus tend to overpopulate the regions of high energies if compared to Maxwell-Boltzmann statistics for phonons. Moreover, as they are localized, it seems that they can deliver more easily the required energy to break bonds. The sum of this facts, i.e., localization, much higher number of breathers above a given activation energy and apparent diminution of the activation energy suggests that DBs are good candidates to explain LTRTs.

\section{Acknowledgments}

JFRA and JC acknowledge sponsorship by the Ministerio de Educación y Ciencia, Spain, project FIS2004-01183. MDA, MN and JMT acknowledge sponsorship from the same body, projects MAT2002-03504 and CTQ200405113. JFRA acknowledges the hospitality and the spectra performed at CNRS-LADIR. All the authors acknowledge Prof. R. Livi, from Florence University for useful discussions.

\section{Appendices}

\section{A Phonon statistics}

\section{A.1 Quantum statistics of one oscillator}

A quantum harmonic oscillator with frequency $\omega$, equal to its classical one, has energies $E_{n}=\left(n+\frac{1}{2}\right) \hbar \omega$. If in contact with a thermal bath at temperature $T$, the probability that it has energy $E_{n}$ is given by $P\left(E_{n}\right)=$ $A \exp \left(-\beta E_{n}\right)=A \exp \left(-\beta\left(n+\frac{1}{2}\right) \hbar \omega\right)$, with $\beta=1 / \mathrm{k}_{\mathrm{B}} T, \mathrm{k}_{\mathrm{B}}$ being the Boltzmann constant.

A can be obtained by the normalization condition $\sum_{n=0}^{\infty} P_{n}=A Z=1$, where $Z=\sum_{n=0}^{\infty} \exp \left[-\beta\left(n+\frac{1}{2}\right) \hbar \omega\right]$ is known as the partition function for the oscillator. $Z$ is a geometric series which can be easily summed leading 
to:

$$
Z=\frac{\exp \left(-\beta \frac{\hbar \omega}{2}\right)}{1-\exp (-\beta \hbar \omega)}
$$

Therefore $A=1 / Z$ and

$$
P_{n} \equiv P\left(E_{n}\right)=\frac{\exp \left[-\beta\left(n+\frac{1}{2}\right) \hbar \omega\right]}{Z}=\exp (-\beta n \hbar \omega)[1-\exp (-\beta \hbar \omega)]
$$

Note that $n$ is the excitation number of the oscillator, however, in solid state physics, where $\omega$ is the frequency of a normal mode of the solid, it is customary to speak of $n$ as the number of phonons with frequency $\omega$. Once known $P_{n}$ a number of quantities can be readily calculated. The mean energy $\langle E\rangle=Z^{-1} \sum_{n=0}^{\infty}\left(n+\frac{1}{2}\right) \hbar \omega \exp \left[-\beta\left(n+\frac{1}{2}\right) \hbar \omega\right]=-Z^{-1} \partial Z / \partial \beta=$ $-\partial \log (Z) / \partial \beta$, which leads to:

$$
\langle E\rangle=\left(\frac{1}{2}+\frac{1}{\exp (\beta \hbar \omega)-1}\right) \hbar \omega .
$$

Consequently, the mean excitation number or mean number of phonons is:

$$
\langle n\rangle=\frac{1}{\exp (\beta \hbar \omega)-1} .
$$

For high temperatures $\hbar \omega / \mathrm{k}_{\mathrm{B}} T<<1$, the mean energy becomes $\langle E\rangle \simeq$ $\mathrm{k}_{\mathrm{B}} T$ which is the classical mean energy of a harmonic oscillator and $\langle n\rangle \simeq$ $\mathrm{k}_{\mathrm{B}} T / \hbar \omega$.

Of particular importance for the present problem is the cumulative probability $C\left(E_{\mathrm{a}}\right)$, i.e., the probability that the oscillator has energy $E_{\mathrm{a}}$ or higher above the ground state $\hbar \omega / 2$, i.e., that it can deliver the energy $E_{\mathrm{a}}$. Let $m$ be the minimum integer so as $m \hbar \omega \geq E_{\mathrm{a}}$, i.e., $m=\left\lceil E_{\mathrm{a}} / \hbar \omega\right\rceil$, with $\lceil x\rceil$, the ceiling function that rounds $x$ towards plus infinity. Then $C\left(E_{\mathrm{a}}\right)=Z^{-1} \sum_{n=m}^{\infty} \exp \left[-\beta\left(\frac{1}{2}+n\right) \hbar \omega\right]=Z^{-1} \sum_{n=0}^{\infty} \exp \left[-\beta\left(m+\frac{1}{2}+n\right) \hbar \omega\right]$ $=Z^{-1} \exp (-\beta m \hbar \omega) \times \sum_{n=0}^{\infty} \exp \left[-\beta\left(\frac{1}{2}+n\right) \hbar \omega\right]$, therefore:

$$
C\left(E_{\mathrm{a}}\right)=\exp (-\beta m \hbar \omega)=\exp \left(-\beta\left\lceil\frac{E_{\mathrm{a}}}{\hbar \omega}\right\rceil \hbar \omega\right) .
$$

If $m$ is of the order of a few tens, the expression above approaches to the classical expression $C_{\text {class }}\left(E_{\mathrm{a}}\right)=\exp \left(-\beta E_{\mathrm{a}}\right)$. Note, however, that the quantum probability is somewhat smaller. The ratio between the quantum probability and the classical one is between $\exp (-\beta \hbar \omega)$ and 1. For a frequency as the one given here for the phonons in the $\mathrm{K}^{+}$plane $\omega_{0}=3.16 \cdot 10^{13} \mathrm{~s}^{-1}$ and $T=573 \mathrm{~K}$ this ratio is $\exp \left(-\beta \hbar \omega_{0}\right) \approx 0.65$ and 0.81 for $T=1173 \mathrm{~K}$. 


\section{A.2 Normal modes and phonons}

Let us consider a solid in the linear approximation, with $N_{f}$ degrees of freedom, with $N_{f}=3 \times N_{a}$ for a three-dimensional solid with $N_{a}$ atoms. There are $N_{f}$ normal modes with frequencies $\omega_{i}$ and wave numbers $\mathbf{k}_{\mathbf{i}}$, each one equivalent to a independent harmonic oscillator, and therefore, the previous subsection can be applied to it. The properties of the solid are simply the sum of the properties of the isolated oscillators, just adding the subindex $i$ to the formulae in the preceding section and summing up, i.e., the excitation number $\left\langle n_{i}\right\rangle$ of the mode $i$ (or the number of phonons) and the energy of the solid are given by:

$$
\left\langle n_{i}\right\rangle=\frac{1}{\exp \left(\beta \hbar \omega_{i}\right)+1} \quad ; \quad E=\sum_{i=1}^{N_{f}}\left(\left\langle n_{i}\right\rangle+\frac{1}{2}\right) \hbar \omega_{i}
$$

The number of modes $N_{p h}\left(E_{\mathrm{a}}\right)$ with energy larger or equal to $E_{\mathrm{a}}$ above their ground state is

$$
N_{p h}\left(E \geq E_{\mathrm{a}}\right)=\sum_{i=1}^{N_{f}} \exp \left(-\beta\left\lceil\frac{E_{\mathrm{a}}}{\hbar \omega_{i}}\right\rceil \hbar \omega_{i}\right) .
$$

If $E_{\mathrm{a}}$ is about a few tens larger than any $\hbar \omega_{i}$, then we simply have:

$$
N_{p h}\left(E \geq E_{\mathrm{a}}\right) \simeq N_{f} \exp \left(-\beta E_{\mathrm{a}}\right),
$$

which is the classical expression. Again, the ratio between the quantum and classical expressions of $N_{p h}\left(E \geq E_{\mathrm{a}}\right)$ is somewhat smaller than the unity.

Therefore, the cumulative probability $C\left(E_{\mathrm{a}}\right)$ i.e., the fraction of modes with energies greater of equal to $E_{\mathrm{a}}$, becomes:

$$
C\left(E_{\mathrm{a}}\right) \simeq \exp \left(-\beta E_{\mathrm{a}}\right) .
$$

\section{B Breather statistics}

\section{B.1 Breathers with hard on-site potential}

The breather statistics theory developed in Ref. 34 for $2 \mathrm{D}$ breather in a system with hard on-site potential is based is some simple hypotheses, which, in principle, can be fairly general:

1. An established fact is that breathers in two and three dimensions have a minimum energy $\Delta .{ }^{33}$ 
2. The rate of creation of breathers with energy $E, B(E)$, is proportional to $\exp (-\beta E)$, since breathers form from fluctuations through an activation process.

3. The probability per unit time that a breather with energy $E$ is destroyed, $D(E)$, is postulated to be inversely proportional to $(E-\Delta)^{z}$, with $z$ a constant (which means as other constants hereafter that it does not change with the energy $E$ ) that depends on the system. This law is the simplest mathematical expression that takes into account that large breathers have longer lives than smaller ones, with the lifetime of breathers with minimum energy $\Delta$ equal to zero. It has to be considered as an approximation as it is not derived from first principles.

Let $P_{\mathrm{b}}(E) \mathrm{d} E$ be the probability of existence (or the mean fraction) of breathers with energy between $E$ and $E+\mathrm{d} E$. The rate of destruction of breathers with energy $E$ is proportional to $D(E)$ and $P_{\mathrm{b}}(E)$, therefore, $\exp (-\beta E)=A P_{\mathrm{b}}(E)(E-\Delta)^{-z}, A$ being a constant, or, $A P_{\mathrm{b}}(E)=(E-$ $\Delta)^{z} \exp (-\beta E)$. Since $\int_{\Delta}^{\infty} P_{\mathrm{b}}(E) \mathrm{d} E=1, A$ can be obtained using the change of variable $y=\beta(E-\Delta)$ :

$$
\begin{aligned}
A= & \int_{\Delta}^{\infty}(E-\Delta)^{z} \exp (-\beta E)= \\
& \frac{\exp (-\beta \Delta)}{\beta^{z+1}} \int_{0}^{\infty} y^{z} \exp (-y) \mathrm{d} y=\exp (-\beta \Delta) \beta^{-(z+1)} \Gamma(z+1),
\end{aligned}
$$

with $\Gamma(z+1)=\int_{0}^{\infty} y^{z} \exp (-y) \mathrm{d} y$, the Gamma function. Thus, the probability of breathers with energy between $E$ and $E+\mathrm{d} E$ is given by:

$$
\begin{array}{r}
P_{\mathrm{b}}(E)=\frac{1}{A}(E-\Delta)^{z} \exp (-\beta E)=\frac{(E-\Delta)^{z} \exp (-\beta E)}{\exp (-\beta \Delta) \beta^{-(z+1)} \Gamma(z+1)}= \\
\frac{\beta^{z+1}}{\Gamma(z+1)}(E-\Delta)^{z} \exp [-\beta(E-\Delta)] .
\end{array}
$$

The mean energy is given by:

$$
\begin{array}{r}
\langle E\rangle=\int_{\Delta}^{\infty} E P_{\mathrm{b}}(E) \mathrm{d} E=\Delta+\int_{\Delta}^{\infty}(E-\Delta) P_{\mathrm{b}}(E) \mathrm{d} E= \\
\Delta+\int_{\Delta}^{\infty} \frac{\beta^{z+1}}{\Gamma(z+1)}(E-\Delta)^{z+1} \exp [-\beta(E-\Delta)] \mathrm{d} E= \\
\Delta+\frac{1}{\beta \Gamma(z+1)} \int_{0}^{\infty} y^{z+1} \exp (-y) \mathrm{d} E=\Delta+\frac{\Gamma(z+2)}{\beta \Gamma(z+1)}= \\
\Delta+(z+1) \mathrm{k}_{\mathrm{B}} T .
\end{array}
$$


The cumulative probability $C_{\mathrm{b}}(E)$, i.e., the probability that a breather has energy higher than $E$, is given by:

$$
\begin{aligned}
C_{\mathrm{b}}(E)= & \int_{E}^{\infty} P_{\mathrm{b}}(E) \mathrm{d} E=\int_{E}^{\infty} \frac{\beta^{z+1}}{\Gamma(z+1)}(E-\Delta)^{z} \exp [-\beta(E-\Delta)] \mathrm{d} E= \\
& \frac{1}{\Gamma(z+1)} \int_{\beta(E-\Delta)}^{\infty} y^{z} \exp (-y) \mathrm{d} y=\frac{\Gamma(z+1, \beta(E-\Delta))}{\Gamma(z+1)}, \quad \text { (B-4) }
\end{aligned}
$$

where $\Gamma(z+1, x)=\int_{x}^{\infty} y^{z} \exp (-y) \mathrm{d} y$ is the first incomplete Gamma function. ${ }^{35}$

The energy for which the probability is maximum is given by $E\left(P_{\max }\right)=$ $\Delta+z \mathrm{k}_{\mathrm{B}} T$, which shows that breathers tend to populate higher energies than phonons. As an example, Fig. 11] shows $P_{\mathrm{b}}(E)$ and $C_{\mathrm{b}}(E)$ for breathers with $\Delta=20 \mathrm{~kJ} / \mathrm{mol}$ and $z=2$ and the equivalent magnitudes for phonons. The large energies the larger values of $C_{\mathrm{b}}(E)$ soon compensate for the much smaller number of breathers than phonons (around $10^{-3}$ ). Although the extrapolation of $C_{\mathrm{b}}(E)$ to large energies has to be done with caution and a more elaborate theory has yet to be developed, the basic fact that breathers tend to populate higher energies than phonons can be accepted. Piazza et $\mathrm{al}^{34}$ have succeeded in fitting the cumulative probability in Eq. B-4 with the observed one in numerical experiments, which proves that the hypotheses above are reasonable.

\section{B.2 Breathers with maximum energy}

Hereafter we modify slightly their theory developed above. The on-site potential for the $2 \mathrm{D}$ system in Ref. 34 is hard, i.e., the frequency of the isolated oscillators increases with the frequency, with the consequences that the breather frequency lies above the phonon band, its energy increases with its frequency and can be considered as unbounded.

For soft on-site potentials or, as in the present paper, potentials with both soft and hard parts, breather energies may have an upper limit because the breather frequency enters the phonon band or because a bifurcation where the breather disappears or transforms into a different one, as a multibreather or a breather with different symmetries. The changes are obtained by introducing an upper limit $E_{\mathrm{M}}$ in the integrals with respect to the energy. Then, Eq. (B-1) becomes (with $y=\beta(E-\Delta)$ ):

$$
\begin{array}{r}
A=\int_{\Delta}^{E_{\mathrm{M}}}(E-\Delta)^{z} \exp (-\beta E)=\frac{\exp (\beta \Delta)}{\beta^{z+1}} \int_{0}^{\beta\left(E_{\mathrm{M}}-\Delta\right)} y^{z} \exp (-y) \mathrm{d} y= \\
\exp (\beta \Delta) \beta^{-(z+1)} \gamma\left(z+1, \beta\left(E_{\mathrm{M}}-\Delta\right)\right)(B-
\end{array}
$$



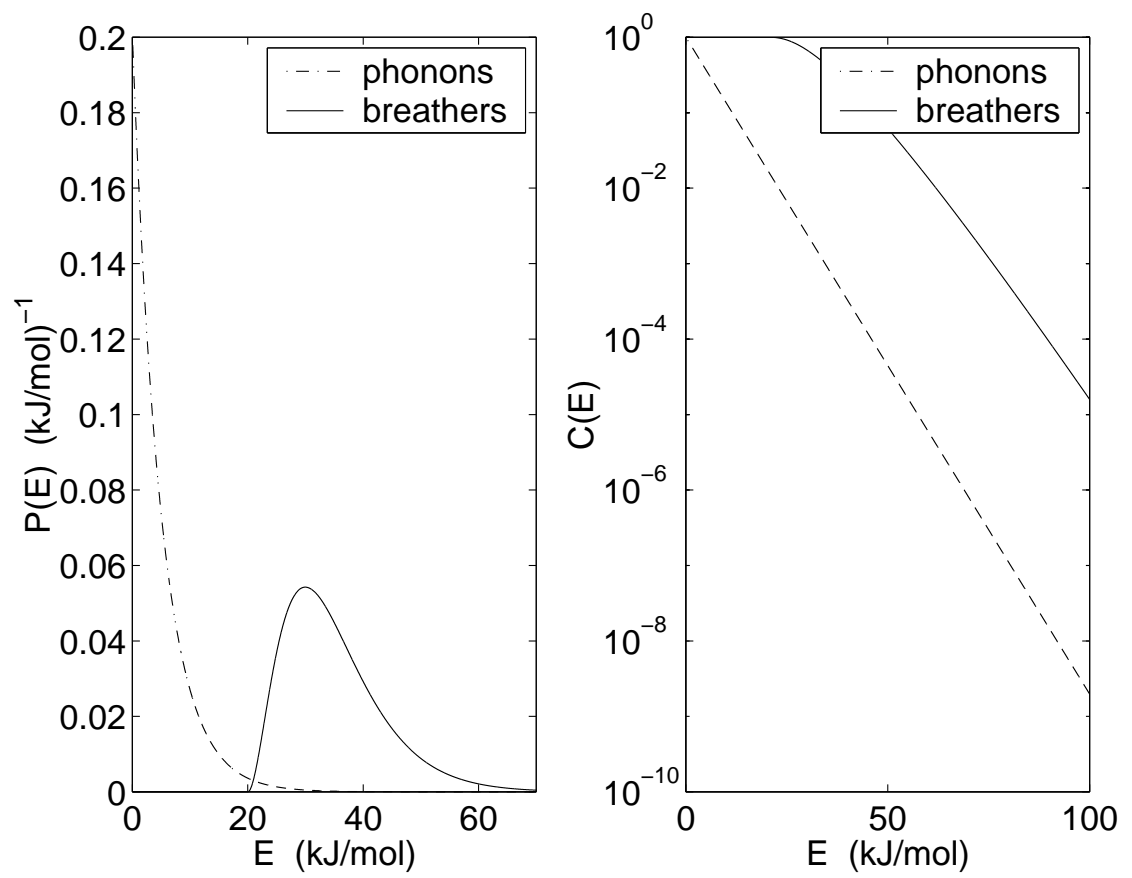

Figure 11: Comparison of the phonon and breather probabilities densities (left) and cumulative probabilities (right). The breather values have been obtained for $z=2$ and $\Delta=20 \mathrm{~kJ} / \mathrm{mol}$. The temperature is $T=600 \mathrm{~K}$ and $\mathrm{k}_{\mathrm{B}} T \approx 5 \mathrm{~kJ} / \mathrm{mol}$.

where $\gamma(z+1, x)=\int_{0}^{x} y^{z} \exp (-y) \mathrm{d} y$ is the second incomplete gamma function. ${ }^{35}$

Therefore, the probability density becomes:

$$
P_{\mathrm{b}}(E)=\frac{1}{A}(E-\Delta)^{z} \exp (-\beta E)=\frac{\beta^{z+1}(E-\Delta)^{z} \exp [-\beta(E-\Delta)]}{\gamma\left(z+1, \beta\left(E_{\mathrm{M}}-\Delta\right)\right)} .
$$

The cumulative probability becomes:

$$
\begin{gathered}
C_{\mathrm{b}}(E)=\int_{E}^{E_{\mathrm{M}}} P_{\mathrm{b}}(E) \mathrm{d} E=\int_{E}^{E_{\mathrm{M}}} \frac{\beta^{z+1}(E-\Delta)^{z} \exp [-\beta(E-\Delta)]}{\gamma\left(z+1, \beta\left(E_{\mathrm{M}}-\Delta\right)\right)} \mathrm{d} E= \\
\frac{1}{\gamma\left(z+1, \beta\left(E_{\mathrm{M}}-\Delta\right)\right)} \int_{\beta(E-\Delta)}^{\beta\left(E_{\mathrm{M}}-\Delta\right)} y^{z} \exp (-y) \mathrm{d} y=1-\frac{\gamma(z+1, \beta(E-\Delta))}{\gamma\left(z+1, \beta\left(E_{\mathrm{M}}-\Delta\right)\right)}(\mathrm{B}-7)
\end{gathered}
$$

For $E_{\mathrm{M}}>\Delta$, the expressions above for $P_{\mathrm{b}}(E)$ and $C_{\mathrm{b}}(E)$ transform into the expressions in Eqs. (B-3B-4) 
In a system like ours there are different types of breathers and the probabilities or cumulative probabilities calculated above correspond to each type with different minimum and maximum energies $\Delta$ and $E_{\mathrm{M}}$ (or without maximum energy), and parameter $z$. The total number of breathers and the relative probability of each type are unsolved questions. The latter probably depends on the temperature, the breather energies, the phase space occupied by each breather and its equivalent ones through symmetries, and the breather profile, which might be excited more or less easily by the phonons. 


\section{References}

1. Muñoz-Páez, A.; Alba, M. D.; Castro, M. A.; Alvero, R.; Trillo, J. M. J. Phys. Chem. 1994, 98, 9850.

2. Alba, M. D.; Alvero, R.; Becerro, A. I.; Castro, M. A.; Muñoz-Páez, A.; Trillo, J. M. J. Phys. Chem. 1996, 100, 19559.

3. Alba, M. D.; Becerro, A. I.; Castro, M. A.; Perdigón, A. C. Amer. Mineral. 2001, 86, 115.

4. Becerro, A. I.; Naranjo, M.; Alba, M. D.; Trillo, J. M. J. Mater. Chem. 2003, 13, 1835-1842.

5. Hong, Z. L.; Yoshida, H.; Ikuhara, Y.; Sakuma, T.; Nishimura, T.; Mitomo, M. J. Eur. Ceram. Soc. 2002, 22, 527.

6. Felshe, J. Struc. Bonding 1973, 13, 99-495.

7. MacKay, R. S.; Aubry, S. Nonlinearity 1994, 7, 1623.

8. Schwarz, U. T.; English, L. Q.; Sievers, A. J. Phys. Rev. Lett. 1999, 83, 223.

9. Fleischer, J. W.; Carmon, T.; Segev, M.; Efremidis, N. K.; Christodoulides, D. N. Phys. Rev. Lett. 2003, 90, 023902.

10. Swanson, B. I.; Brozik, J. A.; Love, S. P.; Strouse, G. F.; Bishop, A. R.; Wang, W.; Salkola, M. I. Phys. Rev. Lett. 2000, 84, 741.

11. Trias, E.; Mazo, J. J.; Orlando, T. P. Phys. Rev. Lett. 2000, 84, 741.

12. Marín, J. L.; Eilbeck, J. C.; Russell, F. M. Phys. Lett. A 1998, 248, 225-229.

13. Russell, F. M.; Eilbeck, J. C. Submitted, 2006.

14. Pérez-Maqueda, L. A.; Franco, F.; Avilés, M. A.; Poyato, J.; PérezMaqueda, J. L. Clays and Clay Miner. 2003, 51, 701-708.

15. Corton, F. A.; Wilkinson, G. W. Advanced Inorganic Chemistry; John Wiley and Sons: New York, 5th ed.; 1988 pg 280.

16. Steeds, J. W.; Russell, F. M.; Vine, W. J. Optik 1993, 92, 149-154.

17. Alba, M. D.; Chain, P. Clay and Clay Miner. 2005, 53, 39-46. 
18. Putnis, A. An Introduction to Mineral Sciences; Cambridge U. Press: Cambridge, UK, 1992.

19. Moore, W. J. Physical Chemistry; Longman: London, third ed.; 1962 pages 528-594.

20. Eyring, H.; Polanyi, M. Z. Physik. Chem. 1931, 12, 279.

21. Back, M. H.; Laidler, K. J. Selected Readings in Chemical Kinetics; Elsevier: , 1967.

22. Pelzer, H.; Wigner, E. Z. Physik. Chem. 1932, 15, 445.

23. Evans, M. G.; Polanyi, M. Trans. Faraday Soc. 1935, 31, 875.

24. Eyring, H. J. Chem. Phys. 1935, 3, 107.

25. Wynne-Jones, W. F. K.; Eyring, H. J. Chem. Phys. 1935, 3, 492.

26. Flach, S.; Willis, C. R. Phys. Rep. 1998, 295, 181.

27. Flach, S.; Mackay, Eds., R. S. Physica D 1999, 119, 1-238 Focus issue.

28. Kivshar, S.; Flach, Eds., S. Chaos 2003, 13, 586-799 Focus issue.

29. Peyrard, M. Nonlinearity 2004, 17, R1-R40.

30. Lide, D. R., Ed.; Handbook of Chemistry and Physics; CRC Press: Boca Raton, Florida, USA, 86 ${ }^{\text {th }}$ ed.; 2005-06.

31. Diaz, M.; Farmer, V. C.; Prost, R. Clays Clay Miner. 2000, 48, 433438.

32. Marín, J. L.; Aubry, S. Nonlinearity 1996, 9, 1501.

33. Flach, S.; Kladko, K.; MacKay, R. S. Phys. Rev. Lett. 1997, 78, 1207.

34. Piazza, F.; Lepri, S.; Livi, R. Chaos 2003, 13, 637-645.

35. Abramowitz, M.; Stegun, I. A. Handbook of Mathematical Functions; Dover: New York, 1972.

36. Fillaux, F. Vibrational Spectroscopy and Quantum Localization. In Energy Localization and Transfer; T. Dauxois, A. Litvak-Hinenzon, R. S. M.; Spanoudaki, A., Eds.; World Scientific: Singapore, 2004; Chapter 2 , pages $73-148$. 